\title{
Ajuste de los Calores de Entrada que se Corresponden con los Tiempos de Enfriamiento de la ZAT en Soldadura GMAW Sobre Acero Dúplex 2205 Empleando la Simulación por Elementos Finitos
}

\author{
Juan A. Pozo-Morejón¹, Luis F. Guimaraes de Souza², Thiago Guerra³, E. V. Morales4, Ivani de Souza Bott³, \\ Amado Cruz-Crespo ${ }^{1}$, Osniel Rodríguez Pérez ${ }^{1}$ \\ 1 Universidad Central "Marta Abreu” de Las Villas, Centro de Investigaciones de Soldadura - CIS, Santa Clara, VC, Cuba. \\ 2 Centro Federal de Educação Tecnológica - CEFET/RJ, Rio de Janeiro, RJ, Brasil. \\ 3 Pontificia Universidade Católica do Rio de Janeiro - PUC-Rio, Departamento de Engenharia Química e de Materiais - DEQM, \\ Rio de Janeiro, RJ, Brasil. \\ 4 Universidad Central “Marta Abreu” de Las Villas, Departamento de Física, Santa Clara, VC, Cuba.
}

Recibido: 04 Jun., 2018

Aprobado: 08 Nov., 2018

E-mail: jpozo@uclv.edu.cu (JAPM)
Resumen: En el presente artículo se realiza un ajuste de los calores de entrada que se corresponden con los tiempos de enfriamiento " $\mathrm{t}_{12 / 8}$ " $\mathrm{y}$ " $\mathrm{t}_{8 / 5}$ " de interés, en la ZAT de la soldadura GMAW de una chapa de acero inoxidable dúplex 2205, de $10 \mathrm{~mm}$ de espesor, empleando la simulación por elementos finitos. Para la modelación de la soldadura se empleó una metodología desarrollada previamente, la cual se enriqueció con la incorporación de un factor de corrección del calor aportado, a los elementos finitos por los que pasa la fuente de calor. La metodología, implementada a través de un código en lenguaje APDL, fue validada inicialmente a través de la comparación con resultados de ciclos térmicos experimentales. En el trabajo además se demuestra que, cuando se utiliza el modelo teórico de Rykalin 2D, son más precisos los resultados que relacionan las variables calor de entrada - tiempo de enfriamiento, empleando las propiedades promedio del acero en el rango de temperatura desde la ambiente hasta la de sólido, que los que se obtienen cuando se sustituyen las propiedades a temperatura ambiente.

Palabras-clave: Acero dúplex; Calor de entrada; GMAW; Simulación de soldaduras; Tiempo de enfriamiento.

\section{Heat Inputs Adjustment Corresponding to the HAZ Cooling Times in GMAW on 2205 Duplex Steel Using Finite Element Simulation}

\begin{abstract}
In the present paper, an adjustment of the heat inputs corresponding to the cooling times of interest " $\mathrm{t}_{12 / 8}$ " and " $\mathrm{t}_{8 / 5}$ " in the HAZ of the GMAW of a duplex stainless steel 2205, of $10 \mathrm{~mm}$ thickness, using finite element simulation is made. For simulation, a welding modeling methodology previously developed was used, and it was enriched with the incorporation of a correction factor of the heat transferred to the finite elements by which the heat source passes. The methodology, using a code in APDL language, was initially validated through the comparison with experimental results of thermal cycles. In the paper is also demonstrated that the results of the relation of the heat input and cooling time variables are more accurate when using the Rykalin 2D theoretical model with the average properties of the steel in the range from room to solid temperature, than those obtained when the properties are replaced at room temperature.
\end{abstract}

Key-words: Duplex steel; Heat input; GMAW; Welding simulation; Cooling time.

\section{Introducción}

Desde hace décadas se han desarrollado modelos teóricos para el análisis de la transferencia de calor en uniones soldadas y uno de los más conocido es el de Rykalin 2D, para flujo de calor bidimensional [1-4]. Este modelo presenta actualidad ya que es uno de los empleados para generar la curva del ciclo térmico, en un equipo moderno destinado a la simulación física de la zona afectada térmicamente de soldadura (ZAT), denominado Gleblee 3800 [4]. Entre otras cosas, dicho modelo permite establecer la Ecuación 1, que relaciona el calor de entrada en una soldadura con los tiempos de enfriamiento " $\mathrm{t}_{12 / 8}$ " $\mathrm{y}$ " $\mathrm{t}_{8 / 5}$ " de la ZAT, que influyen en la microestructura y las propiedades de la unión. 


$$
\Delta t=\frac{Q^{2}}{4 \pi \lambda \rho c d^{2}}\left[\frac{1}{(T 2-T o)^{2}}-\frac{1}{(T 1-T o)^{2}}\right]
$$

donde " $\Delta \mathrm{t}$ " representa el tiempo de enfriamiento en un intervalo de interés definido por las temperaturas " $\mathrm{T}_{1}$ " $\mathrm{V}$ " $\mathrm{T}_{2}$ ", si se cumple que $\mathrm{T}_{1}>\mathrm{T}_{2} ;$ " $\mathrm{Q}$ " el calor de entrada de la soldadura (heat input), que representa un por ciento del

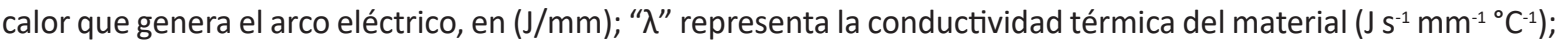
" $\rho c^{\prime}$ el calor específico $\left(\mathrm{J} \mathrm{mm}^{-3} \mathrm{C}^{-1}\right)$; " $\mathrm{d}$ " el espesor de placa $(\mathrm{mm})$; "To" la temperatura inicial de la placa, temperatura de precalentamiento o temperatura entre pasadas $\left({ }^{\circ} \mathrm{C}\right)$.

En ocasiones los especialistas sustituyen en la Ecuación 1 las propiedades físicas del material que se corresponden con la temperatura ambiente, ya que constituyen datos disponibles con facilidad. Por otro lado, esta Ecuación es aplicable solo en el caso que se cumpla la condición de transferencia de calor bidimensional (2D), que se asume ocurre en planos paralelos a la superficie de la placa, en direcciones longitudinal y transversal a la soldadura. En este modelo Rykalin 2D se considera que en cada instante de tiempo la distribución de temperaturas es uniforme a través del espesor de la placa, lo que significa que la configuración de isotermas es idéntica en cualquier plano paralelo a la superficie. Este modelo es utilizable cuando el espesor es inferior a un espesor crítico "dc", que constituye la frontera de aplicación del mismo. Se considera que por encima de "dc" la transferencia de calor de la unión soldada pasa a ser tridimensional, de manera que aparece también la transferencia de calor en dirección normal a la superficie de la placa y el modelo de transferencia en dos dimensiones deja de ser aplicable.

El modelo teórico de Rykalin 2D posee limitaciones, ya que representa una solución extrema de flujo de calor bidimensional en chapa considerada "fina", no puede considerar las variaciones de las propiedades físicas del material respecto a la temperatura y no cuantifica la pérdida de calor al medio, lo que en alguna medida se aleja de lo que acontece en las soldaduras reales.

Estas limitaciones del modelo de Rykalin pueden ser superadas a través del análisis del proceso de transferencia de calor en soldaduras empleando la simulación numérica mediante elementos finitos. Los análisis térmicos de soldaduras que se realizan en este trabajo, a través de un software de elementos finitos de aplicación general, emplean códigos en lenguaje APDL (ANSYS Parametric Design Language) que en esencia se han desarrollado previamente [5-7]. Dichos códigos debe reproducir los aspectos propios de este proceso entre los que se encuentran la geometría y movimiento de la fuente de calor sobre la pieza, el aporte de metal, la transferencia de calor al medio, así como implementar propiedades físicas termodependientes del material.

Los aceros inoxidables dúplex son ampliamente utilizados en aplicaciones de la industria moderna, por lo que la soldadura de estos tiene especial relevancia. Es conocido que en última instancia la estructura y propiedades obtenidas en la ZAT de estos aceros, producto del proceso de soldadura, depende de los tiempos de enfriamiento, " $\mathrm{t}_{12 / 8}$ " $\mathrm{y}$ " $\mathrm{t}_{8 / 5}$ ", que se producen durante el ciclo térmico, en los intervalos de temperaturas de $1200^{\circ} \mathrm{Ca} 800^{\circ} \mathrm{C}$ y de $800^{\circ} \mathrm{C}$ a $500^{\circ} \mathrm{C}$, respectivamente. Estos tiempos de enfriamiento dependen del calor que entra a la unión producto del proceso de soldadura, el que representa un por ciento de la potencia térmica que genera el arco, a partir de los parámetros del régimen utilizados en cada caso. Por tanto, para los especialistas que elaboran procedimientos de soldadura es importante conocer previamente la correspondencia real entre los parámetros que se enumeraron anteriormente, con vistas a conseguir uniones con las propiedades requeridas. Las herramientas con que se cuenta para establecer dicha correspondencia son los modelos teóricos, como el dado en la Ecuación 1, o la simulación mediante elementos finitos que se va imponiendo en las últimas décadas.

En este contexto, el presente estudio se propone como objetivo realizar un ajuste de los calores de entrada de una soldadura real que se corresponden con los tiempos de enfriamiento " $\mathrm{t}_{12 / 8}$ " $\mathrm{y}$ " $\mathrm{t}_{8 / 5}$ " de interés en la ZAT (que barren el rango posible que se presenta industrialmente) de la soldadura GMAW de una chapa de acero duplex 2205, de $10 \mathrm{~mm}$ de espesor, empleando el modelo teórico de Rykalin 2D y la simulación mediante el método de elementos finitos (MEF). Para lograr lo anterior, previamente el trabajo se propone validar el código en lenguaje APDL utilizado para simular la soldadura en este material, así como enriquecerlo con vistas a aumentar su fiabilidad y precisión en los resultados. 


\section{Materiales y Métodos}

\subsection{Propiedades termofísicas del acero dúplex 2205}

En la simulación mediante MEF se emplearon las propiedades físicas termodependientes del acero 2205, reportadas por Xiaojun y Lixing [8] (Figura 1). Solo se introdujo como modificación el aumento del valor de la conductividad térmica por encima de la temperatura de sólido, con vistas a simular la transferencia de calor por convección que se produce en el baño de metal líquido de una soldadura real. De esta manera se logra aproximar la transferencia de calor por convección en el baño líquido utilizando el tradicional modelo sólido de elementos finitos, con transferencia de calor por conducción, mediante el aumento forzado de la conductividad térmica del material, en el rango de temperaturas por encima de la temperatura de fusión. Lo anterior evita la necesidad de simular el movimiento de un fluido, lo que aumentaría innecesariamente la complejidad. En los modelos se asumió una densidad constante del material, de $7800 \mathrm{~kg} \mathrm{~m}^{-3}$ [9], ya que su variación respecto a la temperatura no posee un peso relevante en los resultados.
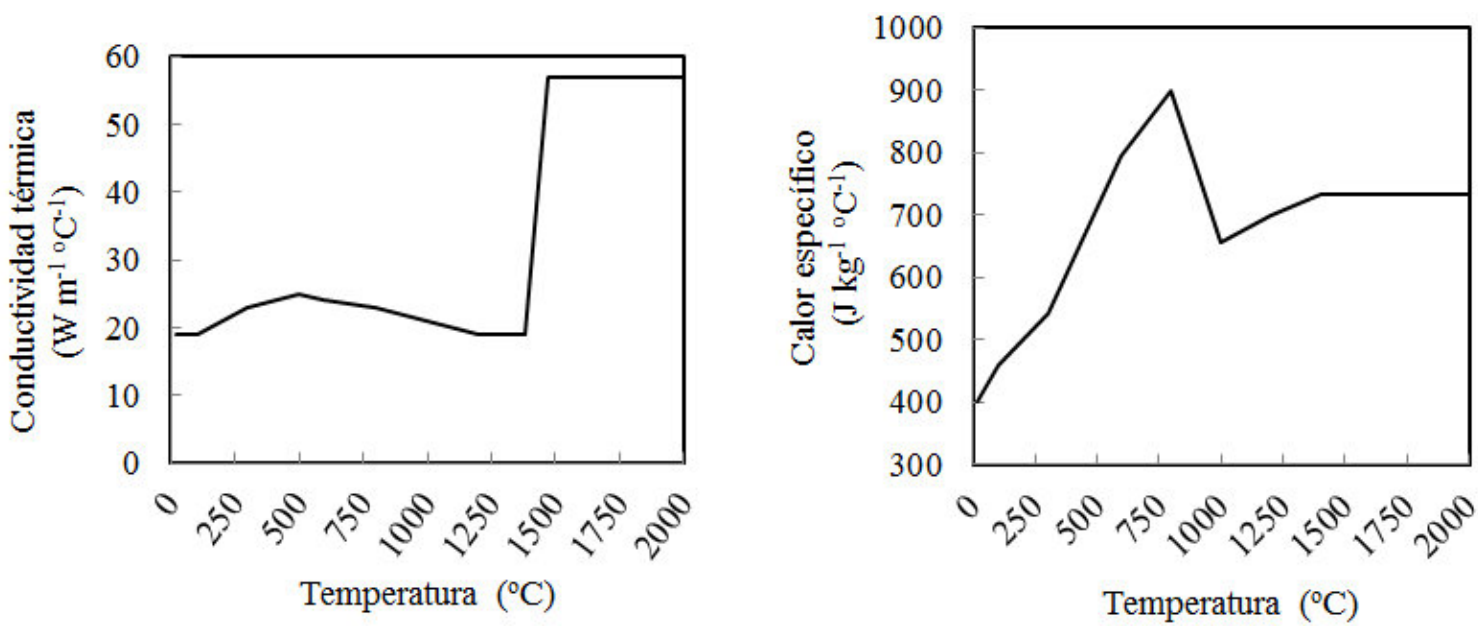

Figura 1. Propiedades físicas termodependientes del acero 2205 empleadas en la simulación.

En el trabajo se utilizaron, cuando se aplica el modelo teórico (Ecuación 1), los valores de propiedades termofísicas constantes para el acero dúplex 2205, en primera instancia las promedio desde la temperatura ambiente hasta la de sólido, determinadas por el método de las áreas bajo las curvas de la Figura $1: \lambda \approx 22 \cdot 10^{-3} \mathrm{~J} \mathrm{~s}^{-1} \mathrm{~mm}^{-1}{ }^{\circ} \mathrm{C}^{-1} ; \rho c \approx 5,3 \cdot 10^{-3} \mathrm{~J} \mathrm{~mm} \mathrm{~mm}^{-3} \mathrm{C}^{-1}[8]$. En segunda instancia, en el modelo teórico se emplearon las propiedades del material que se corresponden con la temperatura ambiente de $28^{\circ} \mathrm{C}$, que se obtienen también a partir de dicha Figura: $\lambda \approx 19 \cdot 10^{-3} \mathrm{~J} \mathrm{~s}^{-1} \mathrm{~mm}^{-1}{ }^{\circ} \mathrm{C}^{-1}$; $\rho c \approx 3,12 \cdot 10^{-3} \mathrm{~J} \mathrm{~mm}^{-3}{ }^{\circ} \mathrm{C}^{-1}[8]$

\subsection{Soldadura experimental}

Con vistas a validar el modelo de elementos finitos se realizó una soldadura GMAW del tipo cordón sobre chapa, de acero inoxidable duplex 2205, con dimensiones de 9,5 × $150 \times 300 \mathrm{~mm}$ (Figura 2). En la soldadura experimental se empleó una fuente de energía inversora y se montó la antorcha de soldadura semiautomática sobre un tractor de oxicorte mecanizado, que garantiza la velocidad de desplazamiento constante previamente definida. La soldadura se realizó con CD, electrodo conectado al positivo. Se empleó una mezcla protectora de $98 \%$ de $\operatorname{Ar}$ y $2 \%$ de $\mathrm{CO}_{2}$, con un flujo de $18 \mathrm{l} / \mathrm{min}$. El material de aporte fue un alambre clasificado como AWS ER2209, de 1,2 mm de diámetro. El régimen de soldeo empleado fue: $\mathrm{Is}=185 \mathrm{~A}, \mathrm{Va}=25,7 \mathrm{~V}, \mathrm{vs}=0,0023 \mathrm{~m} \mathrm{~s}^{-1}$. La soldadura fue ejecutada según Figura 2, comenzando a $5 \mathrm{~mm}$ del borde de la placa, con una longitud de soldeo de $129 \mathrm{~mm}$. 


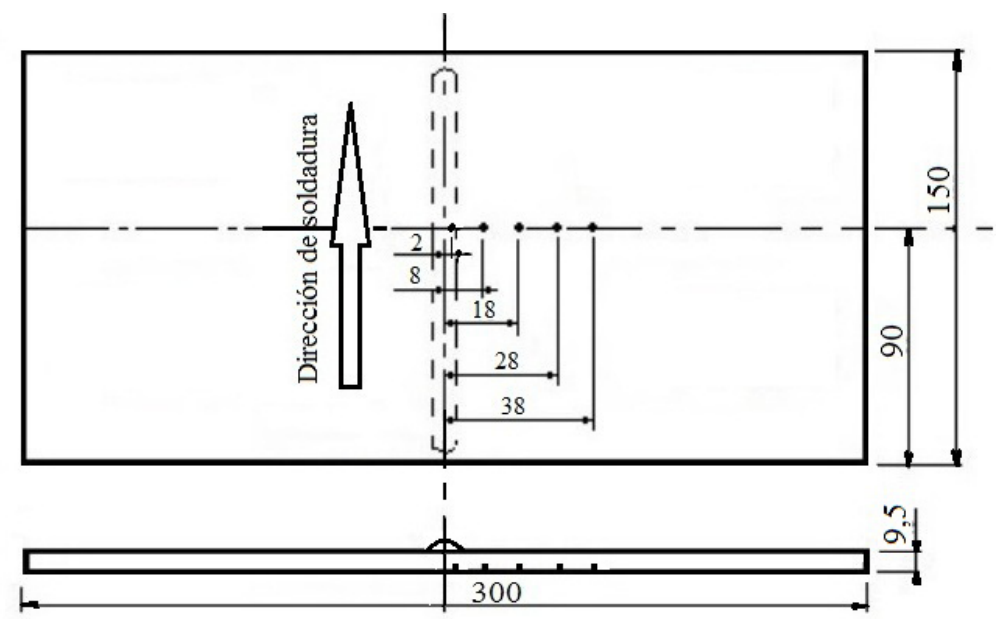

Figura 2. Esquema de soldadura y ubicación de los termopares.

Durante el experimento se llevó a cabo la medición de temperatura mediante cinco termopares tipo " $\mathrm{K}$ ", colocados en el reverso de la chapa e introducidos en agujeros de 1,5 mm de diámetro y profundidad de $4 \mathrm{~mm}$ (Figuras 2 y 3). Dichos termopares fueron colocados a distancias de $2 \mathrm{~mm}, 8 \mathrm{~mm}, 18 \mathrm{~mm}, 28 \mathrm{~mm}$ y $38 \mathrm{~mm}$ del centro del cordón. La señal de los termopares se registró empleando un equipo multicanal de adquisición de datos, con una frecuencia seleccionada de registro de 50 lecturas por segundo.

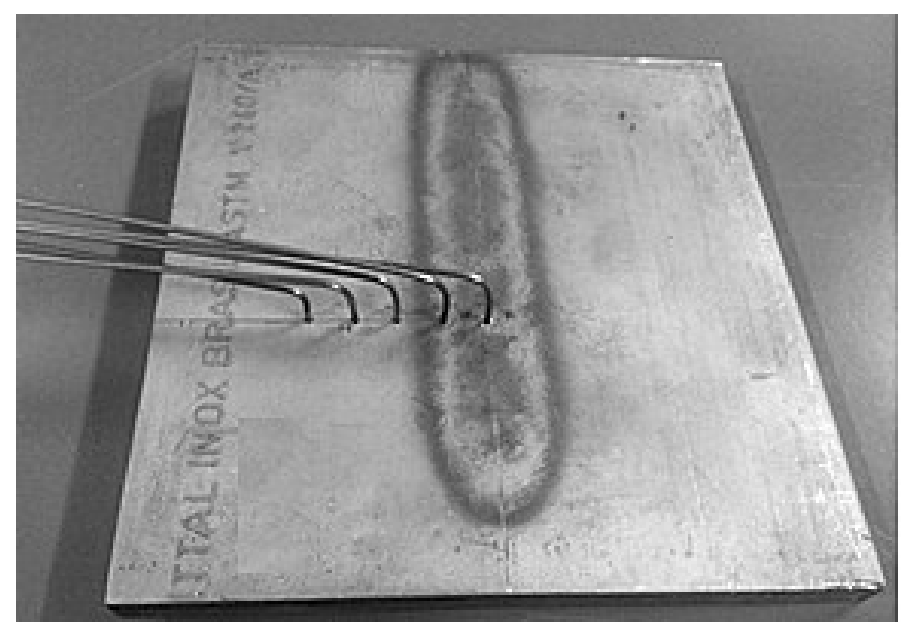

Figura 3. Ubicación de termopares en reverso de placa.

\subsection{Elaboración de modelos para la simulación de soldaduras GMAW sobre chapas de acero 2205 empleando el análisis por elementos finitos}

\subsubsection{Modelo para la validación de análisis térmico por elementos finitos}

El estudio mediante elementos finitos que se realizó en este trabajo partió de la validación del modelo de soldadura GMAW, sobre chapa de acero duplex 2205, a través de la elaboración y corrida del mismo y el posterior cotejo de los resultados de ciclos térmicos simulados, respecto a los obtenidos durante la soldadura real. La modelación fue ejecutada empleando una metodología de análisis térmico de soldaduras por elementos finitos desarrollada previamente [5-7], con la implementación del modelo de fuente de calor de doble elipsoide 
de Goldak [10,11], mediante código en lenguaje APDL. Se ajustó la eficiencia térmica del proceso ( $\eta$ ) a 85\%, que es la que garantizó un menor error relativo entre las curvas de los ciclos térmicos experimentales y simuladas. Este valor se corresponde con el reportado por la mayoría de los autores, que refieren una eficiencia térmica del proceso GMAW entre 0,66 y 0,90 [12-14]. De esta manera se consideró que la potencia térmica proveniente del arco que se introdujo en la pieza (calor de entrada) fue de 4041,3 W.

Desde el punto de vista geométrico, de aplicación de la carga térmica, de pérdida de calor por convección al medio, así como del proceso de transferencia de calor por conducción a través del sólido, el modelo a analizar es simétrico, por lo que se simuló solo la mitad de la pieza, lo que redujo el tiempo computacional. Se construyó el modelo geométrico de la chapa con dimensiones de 9,5 ×150 × $150 \mathrm{~mm}$, con la mitad del cordón de soldadura sobre un borde, con refuerzo de $5 \mathrm{~mm}$ y semiancho de $8,1 \mathrm{~mm}$, que coinciden con dimensiones de soldadura real.

Se empleó en el mallado el elemento SOLID70 de seis caras y ocho nodos [15]. Se refinó la malla en la zona cercana a la soldadura y se hizo más basta en las zonas alejadas. Se utilizaron dos dimensiones de malla, una gruesa, con elementos cúbicos en la zona de la soldadura y cercana, con una dimensión de arista de 1,62 mm (Figura 4) y una fina con 0,81 mm. Esta dimensión de arista en la malla gruesa siguió la recomendación de Goldak [10], de garantizar al menos cinco elementos dentro de la dimensión del semiancho del elipsoide, que se hizo coincidir aproximadamente con el semiancho de la zona fundida determinado experimentalmente.

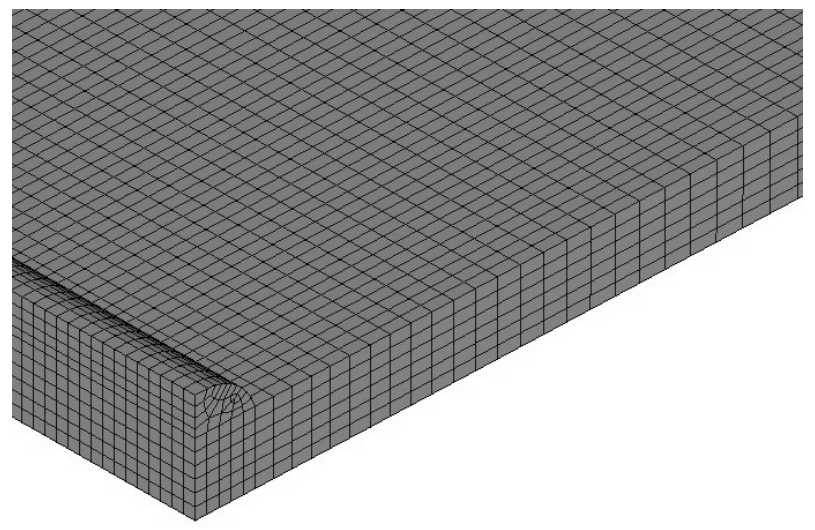

Figura 4. Malla gruesa.

Dentro del tiempo de arco, para cada paso de tiempo, el código APDL calcula la carga térmica que se aplica a cada elemento finito por el que pasa la fuente de calor a través de las expresiones de Goldak para el modelo de elipsoide delantero y trasero, a partir de la distancia del centro de este hasta el centroide de cada elemento [10,11]. En el código se aplica dicha carga térmica a partir del comando "BFE" y la etiqueta "HGEN". Se garantizó el movimiento de la fuente de calor sobre los elementos, a través de toda la longitud de soldadura, con vistas a aplicar la carga térmica al modelo, mediante lazos "*DO - *ENDDO". En cada paso del tiempo de arco se resuelve el sistema y finalizado cada uno se elimina la carga mediante comandos para tales efectos. Para concluir se resuelve un paso de tiempo final, que equivale al tiempo de enfriamiento de la placa hasta la temperatura ambiente, que contiene subpasos de tiempo variable.

Siguiendo la metodología preestablecida, fue empleado el método de solución "full" de "Newton Rapson", un valor de convergencia de la solución de $0,1^{\circ} \mathrm{C}$ y la carga térmica fue aplicada a saltos, en cada paso de tiempo. Como temperatura de partida de la placa, así como del medio circundante, se declaró $28^{\circ} \mathrm{C}$. Se estableció una pérdida de calor por convección al medio, sobre todas las áreas exteriores del modelo, declarando un coeficiente de convección de $12 \mathrm{~W} \mathrm{~m}^{-2}{ }^{\circ} \mathrm{C}^{-1}$, exceptuándose solo el área de simetría que se comporta como adiabática. Solo se consideraron las pérdidas de calor por radiación a través del ajuste del valor de eficiencia de la fuente de calor ( $\eta$ ), como recomiendan varios autores [5-7,16].

Durante el tiempo de arco se empleó un paso de tiempo de $1 \mathrm{~s}$ con vistas a que el centro de la fuente de Goldak salte una dimensión inferior a un cuarto de su longitud, lo que cumple con recomendación de este autor [10]. 
Para el modelo de doble elipsoide fueron establecidas dimensiones cercanas a las de la sección transversal de la zona fundida, según macrografía realizada, adoptándose un semiancho de $9 \mathrm{~mm}$, una profundidad de $5 \mathrm{~mm}$, un semilargo delantero de $9 \mathrm{~mm}$ y uno trasero de $18 \mathrm{~mm}$. El centro del doble elipsoide se desplazó por el centro del cordón, $3 \mathrm{~mm}$ por encima del nivel de la superficie superior de la chapa. Fue establecida una duración de $6000 \mathrm{~s}$ para el paso de tiempo final de enfriamiento, que garantiza el retorno del modelo a una temperatura cercana a la ambiente. De inicio en el modelo se desactivaron los elementos del cordón de soldadura para luego activarlos en el momento en que la fuente se encuentra sobre ellos, mediante comandos para tal efecto.

Como algo novedoso establecido respecto a trabajos precedentes [5-7], se utilizó en el código APDL un coeficiente de corrección del calor que se introduce al modelo por cada elemento finito. Este coeficiente de corrección se determinó mediante el cociente del calor introducido a la pieza por la soldadura real (calculado en Joule a partir de la eficiencia térmica del proceso, intensidad de corriente, voltaje y tiempo de arco) y el calor total que se introduce al modelo (suma del calor en Joule, que se aplica como carga térmica a todos los elementos del modelo, en todos los pasos del tiempo de arco). Lo anterior se logra repitiendo al inicio de la etapa de solución del programa APDL el mismo lazo “*DO - *ENDDO" de aplicación del calor en cada elemento y paso de tiempo, pero en este caso, sin resolver el sistema, eliminando el comando para tal efecto. Finalmente, al ejecutar la simulación definitiva de aplicación del calor al modelo y movimiento de la fuente se empleó un lazo similar al anterior, pero introduciendo el comando de la Ecuación 2, además del comando de solución del sistema en cada paso de tiempo. De esta forma se garantiza que, independientemente del tamaño y número de los elementos finitos de la malla, el calor total que finalmente se introduce al modelo coincida exactamente con el calor que se aporta en la soldadura real, eliminando una posible fuente de error en los mismos.

$$
\text { BFE, ELEMENTO,HGEN, ,QELEM*COEFCOR }
$$

donde "QELEM" representa la densidad de potencia térmica calculada para cada elemento a través de expresión del modelo de doble elipsoide de Goldak [10,11] y "COEFCOR" el coeficiente de corrección referido.

\subsubsection{Modelo de análisis térmico mediante elementos finitos para ajuste de los calores de entrada}

Como es de esperar, debido a las simplificaciones propias del modelo de Rykalin 2D, empleando propiedades termofísicas medias del material entre la temperatura ambiente y de sólido, o simplemente las propiedades a temperatura ambiente, debe existir un error en los calores de entrada que este relacionan con los tiempos de enfriamiento de interés $\left(t_{12 / 8} \mathrm{yt}_{8 / 5}\right)$ en la ZAT. Los tiempos de enfriamiento asumidos como de interés en el estudio, se muestran en las columnas sin resaltar de la Tabla 1 y se determinaron a través de la Ecuación 1 o modelo de Rykalin, a partir de calores de entrada seleccionados de 1000, 1500, 2000, 2500 y $3000 \mathrm{~J} \mathrm{~mm}^{-1}$, de manera que barren el rango que se presenta durante la soldadura industrial GMAW de estos. De esta forma los calores de entrada se ajustaron realizando corridas sucesivas del modelo de elementos finitos para diferentes valores de estos, hasta llegar a los que produjeron los tiempos de enfriamiento más cercanos a los de interés.

Tabla 1. Calores de entrada de soldadura ajustados, empleando la simulación por elementos finitos, que se corresponden con los tiempos de enfriamiento determinados según modelo teórico de Rykalin 2D.

\begin{tabular}{|c|c|c|c|c|c|c|c|c|c|c|}
\hline & \multicolumn{10}{|c|}{ Calor de entrada $\left(\mathrm{J} \mathrm{mm}^{-1}\right)$} \\
\hline & $\mathbf{R}^{2} \mathrm{D}_{\mathrm{m}}$ & MEF & $R_{2} D_{m}$ & MEF & $\mathbf{R}^{2} \mathbf{D}_{\mathrm{m}}$ & MEF & $\mathbf{R}^{2} \mathbf{D}_{\mathrm{m}}$ & MEF & $\mathbf{R}^{2} \mathrm{D}_{\mathrm{m}}$ & MEF \\
\hline & 1000 & 920 & 1500 & 1350 & 2000 & 1800 & 2500 & 2300 & 3000 & 2900 \\
\hline DR (\%) & & 8,7 & & 11,1 & & 11,1 & & 8,7 & & 3,4 \\
\hline$t_{12 / 8}(s)$ & 6,4 & 5,8 & 14,4 & 12,7 & 25,6 & 25,1 & 40,1 & 41,7 & 57,7 & 62,6 \\
\hline DR (\%) & & 10,3 & & 13,4 & & 2,0 & & 3,8 & & 7,8 \\
\hline $\mathbf{t}_{8 / 5}(\mathbf{s})$ & 18,9 & 21,9 & 42,5 & 50,0 & 75,5 & 80,9 & 117,9 & 116,0 & 169,8 & 156,4 \\
\hline DR (\%) & & 13,7 & & 15,0 & & 6,7 & & 1,6 & & 8,6 \\
\hline
\end{tabular}

R2D representa el modelo teórico de Rykalin 2D, empleando propiedades termofísicas medias del material; MEF representa el moldeo de elementos finitos; DR representa la diferencia relativa entre el calor de entrada del modelo teórico de Rykalin 2D y el de elementos finitos ajustado, así como la diferencia entre los tiempos de enfriamiento de ZAT en ambos modelos. 
Ajuste de los Calores de Entrada que se Corresponden con los Tiempos de Enfriamiento de la ZAT en Soldadura GMAW Sobre Acero Dúplex 2205 Empleando la Simulación por Elementos Finitos

Para el ajuste mediante MEF de los calores de entrada de soldadura GMAW sobre chapa de acero 2205, que constituye el objetivo fundamental del presente trabajo, fue empleada la metodología descrita en el subepígrafe 2.3.1 pero con las particularidades que se declaran a continuación. Se simuló una soldadura de ranura GMAW, que se aproxima a las condiciones del modelo teórico de Rykalin de transferencia de calor en dos dimensiones. Se construyó un modelo de chapa con dimensiones de $10 \times 150 \times 150 \mathrm{~mm}$, que simula la mitad de la unión, aprovechando la simetría del problema. En este caso no se consideró ningún refuerzo de soldadura sobre la superficie de la placa, que solo complicaría innecesariamente el modelo. Para mayor ajuste de los resultados se decidió emplear la malla fina. El centro de la fuente de calor de Goldak se hizo desplazar por un canto de la chapa (que representa el centro de la chapa real de $10 \times 150 \times 300 \mathrm{~mm}$ ), comenzando a $5 \mathrm{~mm}$ del borde y desplazándose una longitud de $138 \mathrm{~mm}$. Como dimensiones geométricas utilizadas en el modelo de doble elipsoide fueron establecidas para el semiancho, la profundidad y el semilargo delantero $7 \mathrm{~mm}$, mientras que para el semilargo trasero se estableció $14 \mathrm{~mm}$. Se utilizó un paso de tiempo de 1s, durante el tiempo de arco.

\section{Resultados y Discusión}

\subsection{Validación del modelo de simulación de soldadura mediante elementos finitos}

Una vez ejecutadas las corridas térmicas con los dos tamaños de malla y siguiendo la metodología descrita en epígrafe 2.3.1 se pudo constatar que las dimensiones de las zonas fundidas son similares, según se aprecia en la Figura 5, con un error relativo entre las profundidades de dicha zona de 4,63\%. La isoterma de la frontera de la zona fundida y las dimensiones de dicha zona fueron establecidas a partir de las temperaturas nodales obtenidas en el modelo. Se construyeron los ciclos térmicos para los nodos colocados en los puntos de ubicación de los termopares del experimento descrito en epígrafe 2.2, los que gráficamente se solaparon completamente. El error relativo entre ambas mallas, de las temperaturas máximas de los ciclos térmicos osciló, de 1,44\% en el nodo ubicado a $2 \mathrm{~mm}$ del centro de la chapa a $0,22 \%$ en el ubicado a $38 \mathrm{~mm}$. De esta manera se corroboró que ambos tamaños de malla son capaces de garantizar precisión en los resultados.
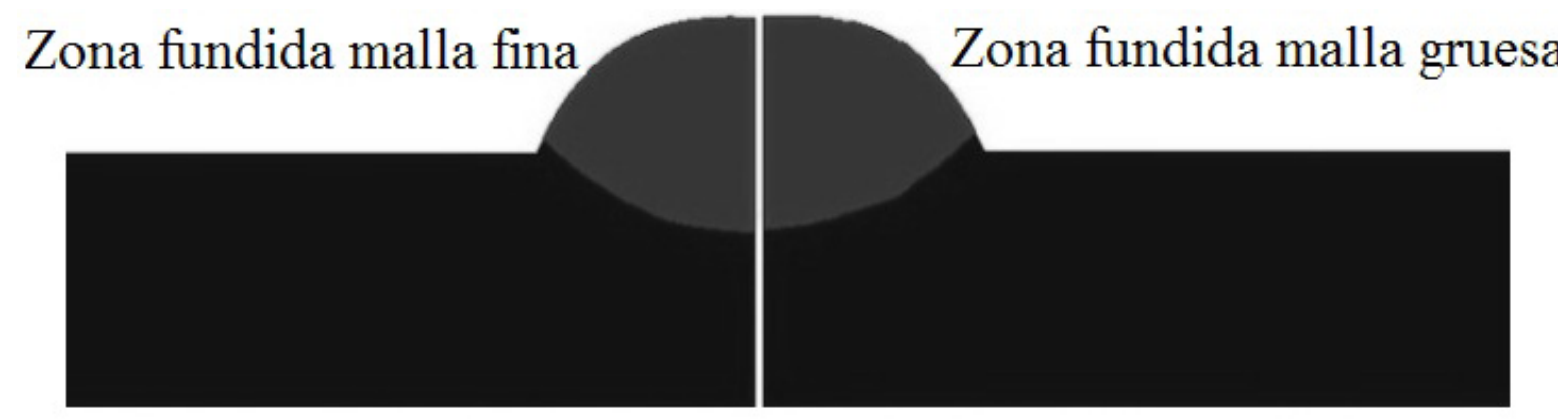

Figura 5. Comparación de la zona fundida predicha por el modelo para ambos tamaños de malla.

Los ciclos térmicos que brinda el modelo fueron superpuestos con los medidos experimentalmente y la coincidencia fue aceptable, con un error relativo para las temperaturas máximas inferior al $5 \%$, salvo en el nodo ubicado a $8 \mathrm{~mm}$ del centro de la soldadura, donde el error fue de 6,9\% (Figura 6). De esta manera se concluye que el código APDL, con el modelo de elementos finitos empleando ambos tamaños de malla y la entrada de datos descrita en epígrafe 2.3.1, garantiza precisión en los resultados, por lo que se considera que el mismo queda validado.

Se corroboró la validez del empleo en el código APDL, de un factor de corrección del calor aportado a cada elemento finito por donde pasa la fuente de Goldak, que garantiza que independientemente de la variación del tamaño de dichos elementos y la cantidad de estos a los que se aplica la carga térmica, se introduzca al modelo la misma cantidad de calor total aportado en la soldadura real, garantizando la precisión de los resultados. 


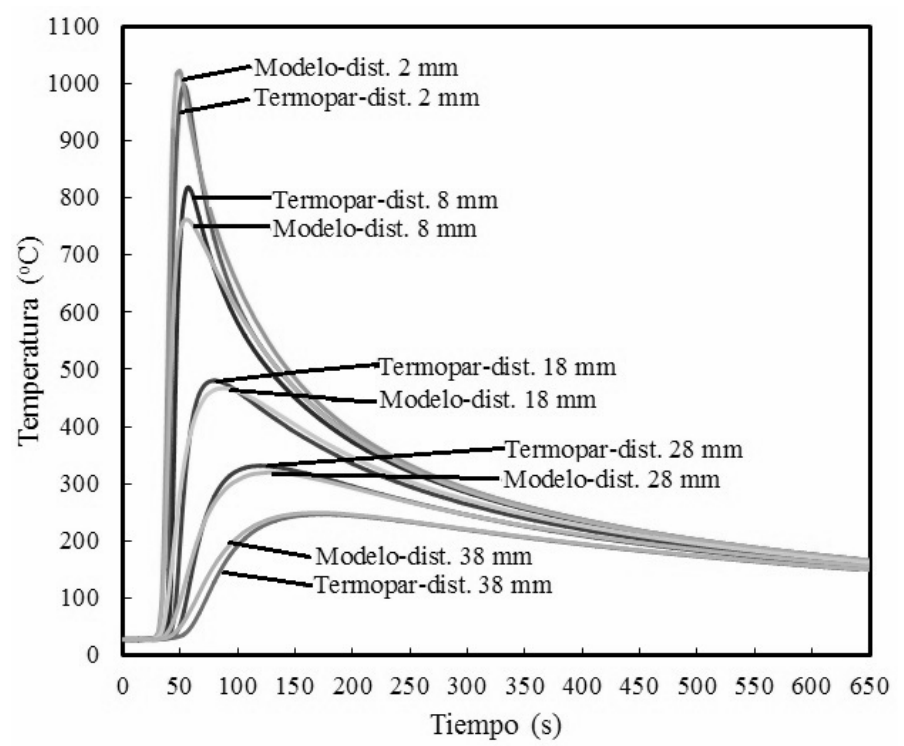

Figura 6. Comparación de ciclos térmicos experimentales y del modelo numérico.

\subsection{Ajuste de los calores de entrada de soldaduras en acero 2205 , que se corresponden con los tiempos de enfriamiento determinados según modelo teórico de Rykalin 2D, empleando la simulación por elementos finitos}

Ya validado el modelo de elementos finitos, se procedió al ajuste mediante simulación numérica de los calores de entrada de soldaduras, que se corresponden con los tiempos de enfriamiento de interés descritos en epígrafe 2.3.1 y que se muestran en la Tabla 1. Para el ajuste de los calores de entrada se empleó el modelo de elementos finitos descrito en subepígrafe 2.3.2.

Los resultados obtenidos mediante MEF se resumen en la Tabla 1, en las columnas resaltadas. Se confirmó que debido a las simplificaciones del modelo de Rykalin 2D, empleando propiedades termofísicas medias del material entre la temperatura ambiente y de sólido, existe un error en los calores de entrada que este relaciona con los tiempos de enfriamiento de interés $\left(\mathrm{t}_{12 / 8} \mathrm{yt}_{8 / 5}\right)$ en la ZAT. Como se muestra en dicha Tabla, para un espesor de chapa a soldar de $10 \mathrm{~mm}$, el calor de entrada de $1000 \mathrm{~J} / \mathrm{mm}$ se ajustó a $920 \mathrm{~J} \mathrm{~mm}^{-1}$, el de $1500 \mathrm{~J} \mathrm{~mm}^{-1}$ se ajustó a $1350 \mathrm{~J} \mathrm{~mm}^{-1}$, el de $2000 \mathrm{~J} \mathrm{~mm}^{-1}$ se ajustó a $1800 \mathrm{~J} \mathrm{~mm}^{-1}$, el de $2500 \mathrm{~J} \mathrm{~mm}^{-1}$ se ajustó a 2300 y el de $3000 \mathrm{~J} \mathrm{~mm}^{-1}$ se ajustó a $2900 \mathrm{~J} \mathrm{~mm}^{-1}$. Para los tres mayores aportes de calor el ajuste de las curvas de enfriamiento de los ciclos térmicos de Rykalin 2D y MEF es alto, dado por diferencias relativas entre los tiempos de enfriamiento $\left(t_{12 / 8} \mathrm{yt}_{8 / 5}\right)$ inferiores a 8,6\% en todos los casos. En los dos menores aportes de calor, el ajuste de las curvas del modelo de Rykalin con el de MEF no es tan alto, ya que las diferencias relativas entre los tiempos de enfriamiento se encuentran entre 10,3 y $15,0 \%$, lo que es un resultado lógico debido a que en estos casos la distribución de temperaturas a través del espesor de la placa se aleja de ser uniforme en la zona cercana a la soldadura y la transferencia de calor comienza a alejarse del modelo en dos dimensiones. Como se observa, todos los calores ajustaron a valores inferiores al del modelo de Rykalin, pero siempre con diferencias relativas entre el modelo teórico y el de MEF inferiores a 11,1\%, lo que indica que este modelo teórico brinda resultados con una precisión aceptable.

Este ajuste de la relación entre los tiempos de enfriamiento de la ZAT y los calores de entrada de la soldadura GMAW, de aceros dúplex 2205 de $10 \mathrm{~mm}$ de espesor, permitirá a los especialistas el establecimiento de regímenes de soldadura que garanticen aquellos tiempos que provocan la obtención de microestructuras y una resistencia a la corrosión y propiedades mecánicas favorables. Empleando este mismo procedimiento se podrían ajustar los calores de entrada que se corresponden con otros tiempos de los ciclos de enfriamiento, para la soldadura de cualquier espesor de chapa de interés, lo que ratifica al MEF como una potente y confiable herramienta para tales efectos.

La Tabla 2 muestra las diferencias relativas entre los calores de entrada ajustados mediante elementos finitos y los predichos por el modelo teórico de Rykalin 2D, empleando en dicho modelo teórico, primero las propiedades 
termofísicas medias del acero 2205 y luego las propiedades a temperatura ambiente, descritas en epígrafe 2.1, que se corresponden con los tiempos de enfriamiento de interés. De dicha Tabla 2 se desprende que los calores de entrada que relaciona el modelo de Rykalin empleando las propiedades medias, son más precisos, con una diferencia relativa inferior a $11,1 \%$, mientras que cuando se emplean las propiedades a temperatura ambiente (que constituye la tendencia de los especialistas al trabajar con la Ecuación 1) la diferencia relativa se hace muy superior, con valores entre 24,4 y $29,3 \%$, que resultan inaceptables por la magnitud del error que provoca. De esta manera se concluye que cuando se pretenda emplear el modelo teórico de Rykalin para calcular los tiempos de enfriamiento en la ZAT, se deben sustituir las propiedades del material medias entre la temperatura ambiente y la de sólido, con vistas a garantizar mayor precisión en los resultados.

Tabla 2. Diferencia relativa del calor de entrada ajustado por elementos finitos respecto a los calores de entrada teóricos determinados mediante modelo teórico de Rykalin 2D, empleando las propiedades físicas del material medias y a temperatura ambiente.

\begin{tabular}{|c|c|c|}
\hline & $\begin{array}{l}\text { Calor de entrada } \\
\left(\mathrm{J} \mathrm{mm}^{-1}\right)\end{array}$ & $\begin{array}{l}\text { DR } \\
\text { (\%) }\end{array}$ \\
\hline MEF & 920 & \\
\hline $\mathrm{R} 2 \mathrm{D}_{\mathrm{m}}$ & 1000 & 8,7 \\
\hline $\mathrm{R} 2 \mathrm{D}_{\mathrm{ta}}$ & 680 & 26,1 \\
\hline MEF & 1350 & \\
\hline $\mathrm{R} 2 \mathrm{D}_{\mathrm{m}}$ & 1500 & 11,1 \\
\hline $\mathrm{R} 2 \mathrm{D}_{\mathrm{ta}}$ & 1020 & 24,4 \\
\hline MEF & 1800 & \\
\hline $\mathrm{R} 2 \mathrm{D}_{\mathrm{m}}$ & 2000 & 11,1 \\
\hline $\mathrm{R} 2 \mathrm{D}_{\mathrm{ta}}$ & 1360 & 24,4 \\
\hline MEF & 2300 & \\
\hline $\mathrm{R} 2 \mathrm{D}_{\mathrm{m}}$ & 2500 & 8,7 \\
\hline $\mathrm{R} 2 \mathrm{D}_{\mathrm{ta}}$ & 1680 & 27,0 \\
\hline MEF & 2900 & \\
\hline $\mathrm{R} 2 \mathrm{D}_{\mathrm{m}}$ & 3000 & 3,4 \\
\hline $\mathrm{R} 2 \mathrm{D}_{\mathrm{ta}}$ & 2050 & 29,3 \\
\hline
\end{tabular}

R2 $D_{m}$ representa el modelo teórico de Rykalin 2D, empleando propiedades termofísicas medias del material; R2D representa el modelo teórico de Rykalin 2D, empleando propiedades termofísicas a temperatura ambiente del material; MEF representa el moldeo de elementos finitos; DR representa la diferencia relativa entre el calor de entrada ajustado mediante elementos finitos respecto al calor calculado por modelo de Rykalin 2D.

\section{Conclusiones}

Los resultados obtenidos en las simulaciones de soldadura mediante elementos finitos, de temperaturas de ciclos térmicos para puntos situados a diferentes distancias del centro de la chapa, manifiestan una alta correlación con los experimentales, con errores relativos inferiores al 7\%, lo cual evidencia la adecuada selección de las propiedades físicas para el acero 2205 y la validez de la metodología y código APDL utilizado para la ejecución del análisis térmico.

Los calores de entrada, que según el modelo teórico de Rykalin 2D se corresponden con los tiempos de enfriamiento de interés, $\mathrm{t}_{12 / 8} \mathrm{yt}_{8 / 5}, \mathrm{y}$ que se determinaron empleando las propiedades físicas constantes medias, entre las correspondientes a la temperatura ambiente y de sólido de la aleación para el acero 2205, son los que mejor se ajustan a los de la zona térmicamente afectada de una soldadura real. Las diferencias relativas calculadas para los aportes de calor ajustados por modelo de elementos finitos y los obtenidos mediante el modelo de Rykalin 2D fueron inferiores al 11,1\% cuando se emplearon las propiedades medias, lo que constituye un error aceptable, y llegaron hasta $29,3 \%$ cuando se sustituyeron propiedades a temperatura ambiente, lo que resulta inaceptable. 
Se concluye que el modelo teórico de Rykalin 2D sigue teniendo actualidad y garantiza una adecuada precisión, cuando se sustituyen las propiedades del material medias entre la temperatura ambiente y la de sólido, con vistas a predecir las velocidades de enfriamiento en la ZAT.

Se ajustaron empleando la simulación por elementos finitos, para los tiempos de enfriamiento de interés en la ZAT, $\mathrm{t}_{12 / 8} \mathrm{yt}_{8 / 5}$, considerando la soldadura de un espesor de chapa de $10 \mathrm{~mm}$ en acero dúplex 2205, los calores de entrada de $1000 \mathrm{~J} \mathrm{~mm}^{-1}$ a $920 \mathrm{~J} \mathrm{~mm}^{-1}$, de $1500 \mathrm{~J} \mathrm{~mm}^{-1}$ a $1350 \mathrm{~J} \mathrm{~mm}^{-1}$, de $2000 \mathrm{~J} \mathrm{~mm}^{-1}$ a $1800 \mathrm{~J} \mathrm{~mm}^{-1}$, de $2500 \mathrm{~J} \mathrm{~mm}^{-1}$ a $2300 \mathrm{~J} \mathrm{~mm}^{-1}$ y de $3000 \mathrm{~J} \mathrm{~mm}^{-1}$ a $2900 \mathrm{~J} \mathrm{~mm}^{-1}$. El ajuste de la relación calor de entrada - tiempo de enfriamiento de la ZAT, en el rango de utilidad industrial, permitirá a los especialistas el establecimiento de los parámetros del régimen de soldadura GMAW que garantizan elevadas resistencia a la corrosión y propiedades mecánicas, evitando aquellos que provocan uniones con propiedades disminuidas.

\section{Agradecimientos}

Los autores del trabajo agradecen a CAPES de Brasil por el financiamiento para la realización de la presente investigación, a través del proyecto No. 88881064968/2014-01, titulado "Influencia de los ciclos térmicos en la evolución microestructural de aceros inoxidables para aplicaciones offshore". Se agradece además, por brindar todo el apoyo necesario, a la Pontificia Universidade Católica do Rio de Janeiro, RJ, Brasil.

\section{Referencias}

[1] Easterling K. Introduction to the physical metallurgy of welding. 2nd ed. Oxford: Butterworth-Heinemann; 1992. 270 p.

[2] Hrivnak I. Theory of weldability of metals and alloys. New York: Ister Science Press; 1992. 372 p.

[3] Poorhaydary K, Patchett BM. Estimation of cooling rate in the welding of plates with intermediate thickness. Welding Journal. 2005;84:149s-155s.

[4] Dynamic Systems, Inc. - DSI. QuikSim2: user's guide release 2.4.5181. Austin: Gleeble System; 2013. 140 p.

[5] Pozo-Morejón JA. Metodología para el establecimiento de procedimientos de soldadura en aleaciones de aluminio al magnesio mediante la modelación por elementos finitos [tesis doctoral]. Santa Clara: Universidad Central "Marta Abreu" de Las Villas; 2008.

[6] Pozo-Morejón JA, García Jacomino J, Ramos Morales F, García Rodríguez Y, Cruz Crespo A, Díaz Cedré E, Duffus Scott A. Metodología de modelación mediante ANSYS de la historia térmica, tensiones y deformaciones de soldadura. Revista de la Facultad de Ingeniería U.C.V. 2009;24(1):13-26.

[7] Pozo-Morejón JA, Quintero-Acosta PE, Cruz-Crespo A, DíazCedré E. Análisis térmico de soldadura GTAW sobre placa de acero AISI 316L empleando el método de elementos finitos. Soldagem e Inspeção. 2011;16(3):256-264. http://dx.doi. org/10.1590/S0104-92242011000300007.
[8] Xiaojun J, Lixing H. Finite element analysis of modeling residual stress distribution in all position duplex stainless steel welded pipe. Materials Science and Technology. 2004;20(4):387-390.

[9] Sandvik. Sandvik SAF 2205: tube and pipe, seamless. 2015 [acceso el 26 junio 2015]. Disponible: www.smt.sandvik. com/en/materials-center/material-datasheets/tube-andpipe-seamless/sandvik-saf-2205/?pdf=1

[10] Goldak JA, Akhlaghi M. Computational welding mechanics. New York: Springer Science+Business Media; 2005. $321 \mathrm{p}$

[11] Nguyen NT, Mai YW, Simpson S, Ohta A. Analytical approximate solution for double ellipsoidal heat source in finite thick plate. Welding Journal. 2004;83:82s-93s.

[12] International ASM. Welding, brazing, and soldering. Materials Park: International ASM; 1993. 2873 p. (ASM Handbook; 6).

[13] American Welding Society-AWS. Welding science and technology. 9th ed. Miami: AWS; 2001. (Welding Handbook; 1).

[14] Saquib M, Untawale SP. Measuring the process efficiency of controlled welding processes. International Journal of Instrumentation. Controle \& Automação. 2012;1(3-4):33-39.

[15] Ansys Inc. Tutorial de Ansys Multiphysics versión 16.1. Pensilvânia: Ansys Inc.; 2015.

[16] Armentani E, Esposito R, Sepe R. Valutazione numérica delle tensioni residue in processi di saldatura GMAW. In: XXXV Convegno Nazionale, Associazione Italiana per l'Analisi delle Sollecitazioni (AIAS); 2006 Settembre 13-16; Roma. Roma: Università Politecnica delle Marche; 2006. 